\title{
Comparative Effect of Cannabis, Aspirin and Cataflam on Nociception in Cd1 Mice
}

\author{
C. O. Nku, G. O. Oghale, I. O. Ajiwhen \\ Department of Human Physiology, College of Medical sciences, University of Calabar, Calabar. PMB 1115, \\ Nigeria.
}

\begin{abstract}
The effects of cataflam, aspirin and the ethanolic extract of Cannabis sativa on nociception was studied in albino mice of both sexes. Forty (40) albino mice were divided into four (4) groups of ten (10) each. The control group was given normal saline (p.o). Test group one (1) was given (p.o) cataflam at a dose of $1.5 \mathrm{mg} / \mathrm{kg}$. Test group two (2) was given (p.o) aspirin at a dose of $13.5 \mathrm{mg} / \mathrm{kg}$. Test group three (3) was given the ethanolic extract of Cannabis sativa at a dose of $10 \mathrm{mg} / \mathrm{kg}$. All four (4) groups were given access to normal mice chow and water ad libitum. The hot plate test was used to assess the effects of the test substances on sensitivity to acute thermal pain while the formalin test was used to assess the sensitivity to persistent pain due to peripheral tissue injury and inflammation. The result shows that Cannabis, Cataflam and Aspirin all reduce sensitivity to pain. This is indicated by the reduction in frequency and duration of hind paw licking in both phases of the formalin test $(p<0.05)$. Cataflam and Cannabis may not have any effect on sensitivity to acute thermal pain. There was no significant difference in the latency of jump in the groups treated with Cataflam and Cannabis during the hot plate test compared to control $(p<0.05)$. But they all reduce sensitivity to persistent inflammatory pain.
\end{abstract}

Keywords: Cannabis sativa, Aspirin, Cataflam, nociception, formalin test, hot plate test

\section{Introduction}

Nociception is the neural processes of encoding and processing noxious stimuli. Nociception refers to the peripheral and central nervous system (CNS) processing of information about the internal or external environment, as generated by the activation of nociceptors. Typically, noxious stimuli, including tissue injury, activate nociceptors that are present in peripheral structures and that transmit information to the spinal cord dorsal horn or its trigeminal homologue, the nucleus caudalis. From there, the information continues to the brainstem and ultimately the cerebral cortex, where the perception of pain is generated (1).

Cannabis is an annual, dioecious, flowering herb. It is a genus of flowering plants that includes three species, Cannabis sativa, Cannabis indica, and Cannabis ruderalis. These three species are indigenous to Central Asia, and South Asia.

The different preparation of Cannabis sativa have been used in Asian traditional medicine for treatment of variety of diseases including: inflammation, nausea, headache, diarrhoea, and alopecia (baldness). $(2,3,4)$. In ancient Iranian Avesta medicine, hashish (bhanga) was mixed with wine to deliver anaesthesia $(5,6)$. Research has shown that short term feeding with Cannabis sativa hempseed feeding improves blood lipid and protein profile (7). Cannabis is prescribed in synthetic form for the treatment of Glaucoma, cachexia, nausea and vomiting (8), also in the treatment of pains, muscle spasticity, convulsant activity, insomnia, hypertension, asthma and depression (9).

Cataflam (diclofenac potassium) is a non-steroidal anti-inflammatory drug (NSAID). This medicine works by reducing substances in the body that cause pain and inflammation. Cataflam is used to treat mild to moderate pain and to treat the signs and symptoms of rheumatoid arthritis and osteoarthritis. Cataflam is also used to treat cramping pain in the lower abdomen associated with menstruation (10).

Aspirin (acetylsalicylic acid) is a derivative of salicylic acid. Found in the bark of willow trees, salicylic acid's medicinal benefits and side effects have been known for more than 2000 years. Aspirin was first synthesized by a Bayer Company chemist in the late 19th century. It proved to be far less of a gastric irritant than salicylic acid and was introduced to the marketplace in the spring of 1899. Heralded as a wonder drug, it quickly became one of the most widely used pharmaceutical products in the world. Acetylsalicylic acid is used to relieve pain, fever, and inflammation in various conditions such as lower back and neck pain, the flu, common cold, burns, menstrual pain, headache, migraines, osteoarthritis, rheumatoid arthritis, sprains and strains, nerve pain, toothache, muscle pain, bursitis (inflammation of a bursa, a fluid-filled sac located around joints and near the bones), and following surgical and dental procedures. Aspirin is also used for rheumatic fever in combination with other medications $(11,12)$. 
Aspirin is a known medication for pain. So is Cataflam. Cannabis sativa has long be used traditionally for the alleviation of pain. This research is aimed at comparing the effects of Cannabis sativa, Cataflam and Aspirin on nociception in mice.

\section{Experimental animals}

\section{Materials And Methods}

Swiss white albino mice (male \& female) were obtained from the animal house of the Department of Physiology, University of Calabar. The animals were housed in the Neurobehaviour laboratory of the College of Medical Sciences, University of Calabar. They were kept at room temperature with 12-hour dark/light cycle. The animals were allowed access to food and water ad libitum. The animals were allowed one week to acclimatize before extract administration.

\section{Plant materials and extract preparation}

Fresh leaves of Cannabis sativa were obtained from Obubra local government area of Cross River state. The leaves were washed to remove debris and oven dried at room temperature. The dried leaves were then grinded to fine powder using a manual blender. The powdered leaves was then subjected to maceration (cold extraction) in an extraction jar using ethanol for 72 hours. The crude extract was then filtered using Whatmann's filter 1 . The filtrate was evaporated using a hot plate at $40^{\circ} \mathrm{C}$. The pasty concentrate was stored in a refrigerator until required for use.

\section{Drug treatment}

Cataflam and Aspirin were purchased from Bez Pharmacy, Calabar and administered at the dosage of $65 \mathrm{mg} / \mathrm{kg}$ and $13.5 \mathrm{mg} / \mathrm{kg}(13,14)$. The ethanolic extract of Cannabis sativa was administered at dose of $10 \mathrm{mg} / \mathrm{kg}$ (15).

\section{Determination of nociception}

Nociception was studied using the formalin test and the hot plate test. Acute thermal pain is modeled by the hot-plate test. Persistent pain produced by peripheral tissue injury and inflammation was studied using the formalin test.

\section{Hot plate test}

The method originally described by Woolfe and MacDonald (16) has been modified by several investigators. Groups of mice of either sex with an initial weight of 18 to $22 \mathrm{~g}$ are used for each dose. The hot plate, which is commercially available, consists of an electrically heated surface. The temperature is controlled for $55^{\circ}$ to $56^{\circ} \mathrm{C}$. This can be a copper plate or a heated glass surface. The animals are placed on the hot plate and the time until either licking or jumping occurs is recorded by a stop-watch. The latency is recorded before and after 20,60 and 90 minutes following oral or subcutaneous administration of the standard or the test compound. The paws of mice and rats are very sensitive to heat at temperatures which are not damaging the skin. The responses are jumping, withdrawal of the paws and licking of the paws. The time until these responses occur is prolonged after administration of centrally acting analgesics, whereas peripheral analgesics of the acetylsalicylic acid or phenyl-acetic acid type do not generally affect these responses.

\section{Formalin test}

The formalin test is used to determine the potential analgesic effects of compounds for states of persistent pain in which tissue damage occurs. The formalin test was carried out in a $30 \times 30 \times 60 \mathrm{~cm}$-sized clear transparent plastic chamber. A mirror placed behind the box allowed for an unobstructed view of the rat's body and the rat's behaviour was recorded on a videotape. The formalin was made of commercially available $37 \%$ formaldehyde solution further diluted in isotonic saline. Conscious rats received a subcutaneous injection of formalin solution into the plantar surface of the right hind paw with a 26-gauge needle. The rats were then placed in an individual cage.

In our analysis, the pain-related behaviors were quantified by determining the incidence of spontaneous flinching of the injected paw or the cumulative time of licking of the injected paw. Flinching is one of the painrelated behaviors in a formalin model and is characterized by spontaneous, rapid, brief shaking or lifting of the paw. Accordingly, each episode of shaking, vibrating or lifting of the paw was counted as one flinch. Flinching and licking were chosen as measures of pain, because they are more spontaneous than other formalin pain related behaviors (e.g. favouring and lifting) and, consequently, are thought to be more reliable for the quantification of the pain-related behaviors. Flinching was counted using the criteria described by WheelerAceto and Cowan (17): reflexive retraction or shaking of the formalin-injected paw, or flinching of the hindquarters, sometimes including most of the body. A nociceptive score was determined for each 5 min block 
by measuring the sum of duration or frequencies of the behaviour. Formalin-induced nociceptive behaviour was assessed in an observer-blind manner. Data were recorded for the early acute phase (phase 1) observed during 0$5 \mathrm{~min}$ after the injection and the late tonic phase (phase 2) observed during 20-60 min after the injection.

The baseline diameters of the hind paws were measured before the formalin injection using a calliper; at the metatarsal level. Those of the hind paws that developed oedema were determined at 4 hours after the injection by measuring the dorsal plantar foot thickness at the metatarsal. Both of the hind paws were measured simultaneously. The 4 hour interval from the formalin injection to the measurement of the paw oedema was set from the literatures for the maximum time to develop an oedema $(18,19)$.

\section{Statistical analysis}

Values for the results were expressed as mean \pm SEM. The statistical analyses were done using the analysis of variance (ANOVA) and the post/hoc Newmann Keul's test. The computer softwares used were Microsoft excel 2007 edition and SPSS 10.0 for windows. Differences between means was considered significant at $\mathrm{P} \leq 0.05$.

\section{Results}

Comparison of latency of jump during the hot plate test in control and test groups.

Fig 1 shows the latency of jumps during the hot plate test. The latency of jump in the group treated with Aspirin is significantly higher $(14.34 \pm 2.25)$ compared to control $(7.50 \pm 1.31)$. However there was no significant difference in the groups treated with Cataflam and Cannabis compared to control. Neither was there a significant difference in the latency of jump between the test groups.

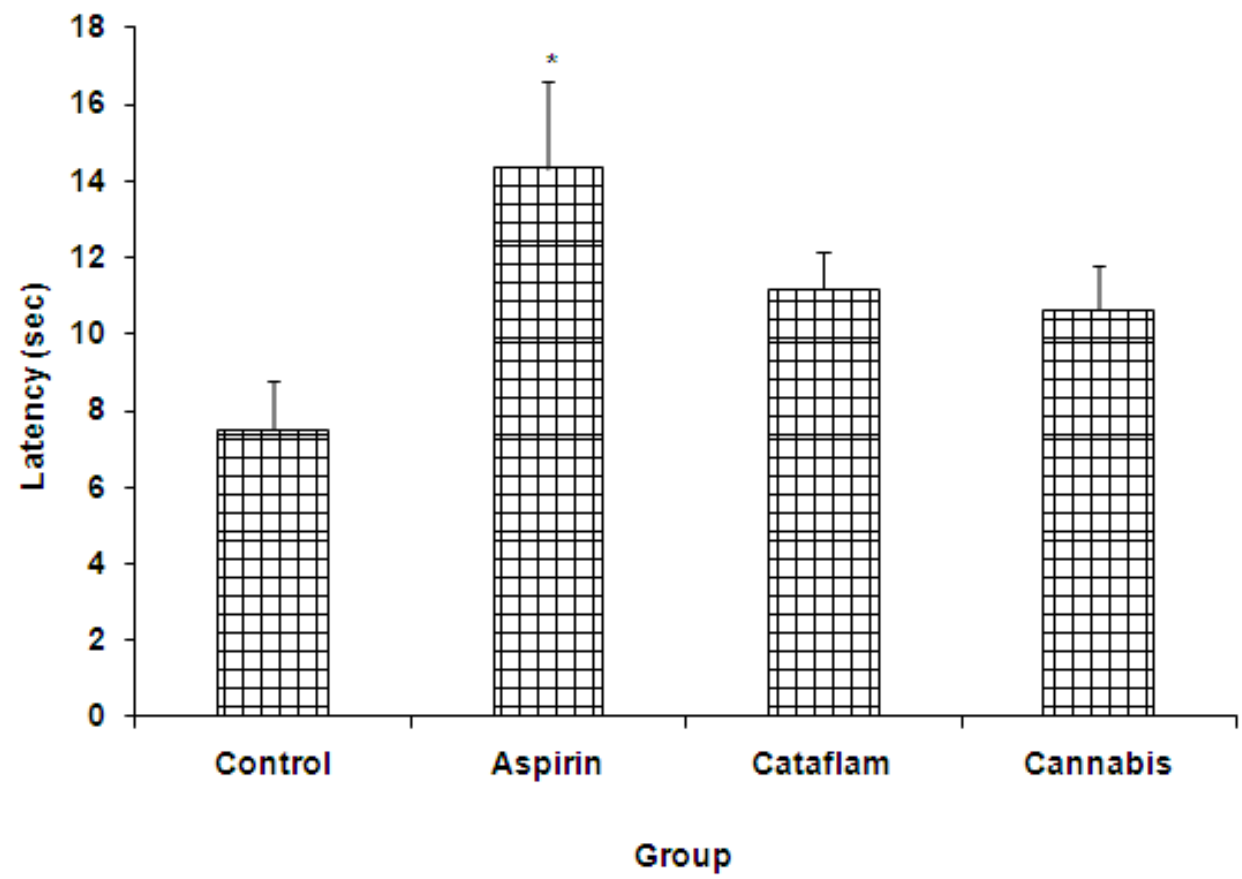

FIGURE 1: Comparison of latency of jump during the hot plate test in control and tests groups. Values are mean $\pm S E M, n=5$. ${ }^{*}$ significantly different from control at $p<0.05$

\section{Comparison of frequency of right hind paw licks in the formalin test.}

Fig 2 compares the frequency of right hand paw licks in the two phases of the formalin test. The frequency of right hand paw lick is significantly lower in all the test groups compared to control in both phases of the formalin test. The frequency is also significantly higher in the Cataflam treated group (16.20 \pm 1.24$)$ compared to the aspirin treated group $(9.20 \pm 1.24)$ in phase 1 . In phase 2 the frequency of right paw licks is significantly higher in the Cannabis treated group compared to the aspirin treated group. 


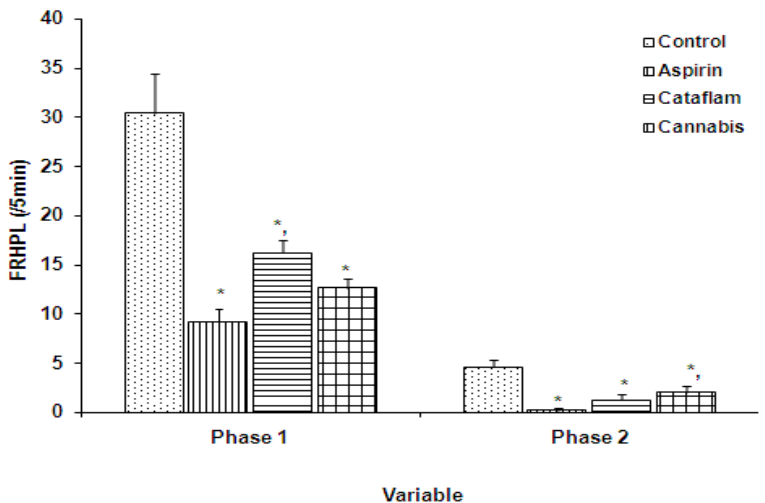

Figure 2: Comparison of frequency of right hind paw licks between the different experimental groups.

*significantly different from control at $p<0.05$

$a=$ significantly different from aspirin at $p<0.05$

\section{Comparison of frequency of left hind paws licks in the formalin test.}

The frequency of left paw lick is significantly lower in the groups treated with aspirin $(15.10 \pm 4.89)$ and Cannabis $(22.14 \pm 4.44)$ compared to control $(48.94 \pm 10.61)$ in phase 1 . There was no significant difference in the frequency of left paw lick between the cataflam treated group and control. In phase 2, the frequency is significantly lower in all test groups compared to control.

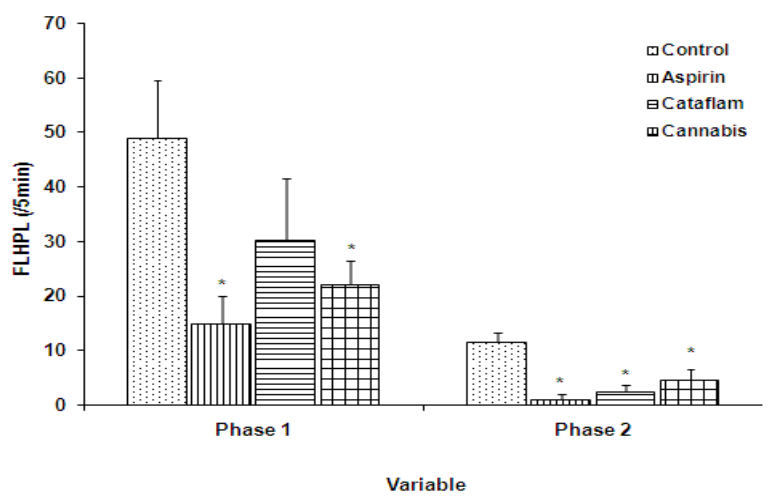

Figure 3: Comparison of frequency of left hind paw licks Values the different experimental groups. significantly different from control at $\mathrm{p}<0.05$
s.

Duration of right hind paw lick in the formalin test

The duration of right hand paw lick is significantly lower in all the test groups compared to control in both phases of the formalin test.

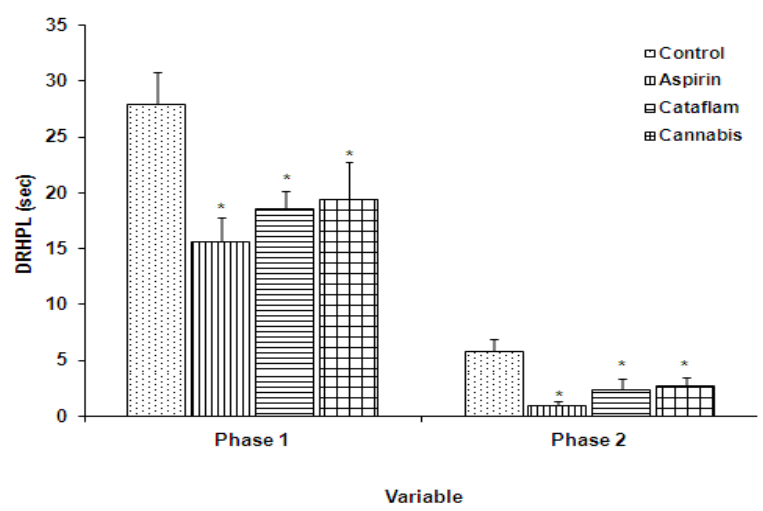

Figure 4: Comparison of duration of right hind paw licks between the different experimental groups.

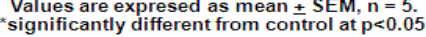




\section{Comparison of duration of left hind paw licks in the formalin test}

The duration is significantly lower in the aspirin and cannabis treated group compared to control. There was no significant difference between the cataflam treated group and the control in phase 1 of the formalin test. In phase 2 the duration was significantly lower in all test groups compared to control.

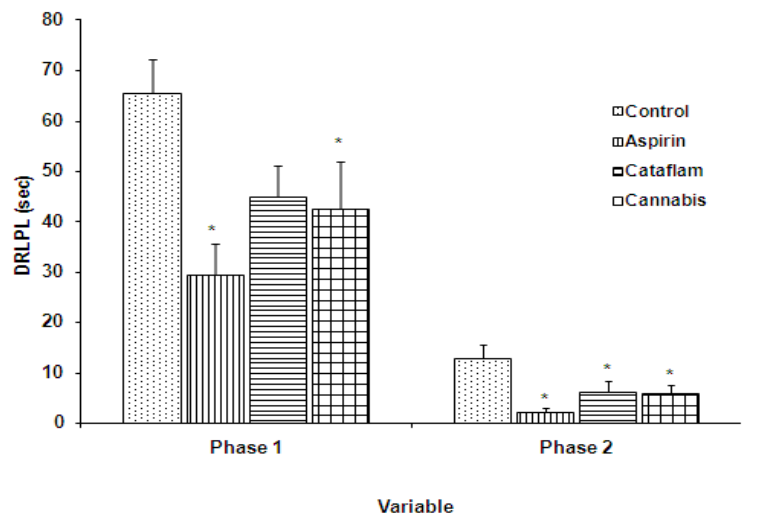

Figure 5: Comparison of duration of left hind paw licks

between the different experimental groups.

Values are expresed as mean $\pm \mathrm{SEM}, \mathrm{n}=5$.
*significantly different from control at $\mathrm{p}<0.05$.

\section{Discussion}

Experimental models of pain sensitivity (nociception) include tests of response thresholds to highintensity stimuli (acute pain tests) and of changes in spontaneous or evoked behavioral responses in animals with peripheral injury or inflammation (persistent pain models). In this research acute thermal pain is studied using the hot-plate test. Persistent pain produced by peripheral tissue injury and inflammation is studied using the formalin test.

The formalin test is used as a model for tonic pain (20) and localized inflammatory pain $(21,22)$. There are two phases of the responses. While the stimulus during the early phase is a direct chemical stimulation of the nociceptors, the stimulus for the late phase involves inflammation (23). It is an interesting aspect of this test that two principally different stimuli are employed in the same test. Formalin-induced pain is caused primarily by peripheral tissue inflammation (24). A central sensitization of dorsal horn neurons occurs during the inflammatory pain. Research by Voilley et al (25) suggests that the combined capacity to block cyclooxygenases and inhibit both inflammation induced expression and activity of acid sensing ion channels (ASICs) present in nociceptors is an important factor in the action of NSAIDs against pain. The results of our experiment shows a uniform reduction in sensitivity to pain (nociception). There was a reduction in sensitivity to pain in the groups treated with aspirin, cataflam and Cannabis (figs. 2-5). This is indicated by the reduction in frequency and duration of hind paw licking in both phases of the formalin test. This agrees with results of previous studies that confirm that these substances have analgesic properties. Edwards et al (26) established the efficacy of aspirin as a post-operative analgesic. A study by Hasani et al (27) shows that Cataflam (Diclofenac) has preemptive and anti-nociceptive effects in acute thermal and inflammatory induced pain. However, when we compared the degree of reduction in the sensitivity to pain by the three test substances (Aspirin, cataflam and Cannabis) we found no significant difference of cannabis compared to aspirin and cataflam. There was a significantly lower frequency of right hind paw lick (fig 2) in the aspirin treated group compared to cannabis which would suggest that aspirin has a greater analgesic effect compared to Cannabis. This is consistent with our result in the hot plate test. In the hot plate test the latency of jump is significantly higher in the aspirin treated group compared to control. There was no significant difference in the latency of the other two groups treated with cataflam and cannabis. This may mean that Cataflam and Cannabis do not have an effect on acute thermal pain. However this is not in agreement with the findings of Hasani et al (27) who observed an anti-nociceptive effect of Diclofenac in Formalin test. Interestingly, there was a significant reduction in duration and frequency of right hind paw lick in the group treated with Diclofenac in phase one of the formalin test. This was not the case with the duration and frequency of paw lick in the left hind paw. Though there seemed to be some reduction, the reduction was not statistically significant. Torres-Lopez et al (28) observed a nociceptive effect with Diclofenac only in the second phase of the formalin test. There was no significant reduction in flinching behavior in the first phase of the formalin test.

In summary, our result shows that Cannabis, Cataflam and Aspirin all reduce sensitivity to pain. This is indicated by the reduction in frequency and duration of hind paw lick in both phases of the formalin test. Cataflam and Cannabis may not have any effect on sensitivity to acute thermal pain. There was no significant 
difference in latency of jump in the groups treated with Cataflam and Cannabis during the hot plate test compared to control $(\mathrm{p}<0.05)$. But they all reduce sensitivity to persistent inflammatory pain.

\section{Conclusion}

Cannabis, Cataflam and Aspirin all reduce sensitivity to pain. Our results show that Cataflam and Cannabis may not have any effect on sensitivity to acute thermal pain. But they all reduce sensitivity to persistent inflammatory pain. The results indicate that aspirin may have the greatest analgesic effect.

\section{References}

[1]. National Academy of Sciences. Recognition and Alleviation of Pain in Laboratory Animals, 2009. National Academies Press.

[2]. McPartland, M.J. Random queries concerning the evolution of Cannabis and coevolution with the cannabinoid receptor. In: Guy. G., Robson, R., Strong, K. and Whittle, B. The Medicinal Use of Cannabis. Royal Society of Pharmacists, London, 2004; pp: 71 102 .

[3]. Xiaozhai, L., Clarke, R. C. The cultivation and use of hemp (Cannabis sativa L.) in ancient China. Journal of the International Hemp. Association 1995; 2: 26-33.

[4]. Zias, J., Stark, H., Sellgman, J., Levy, R., Werker, E., Breuer, A., Mechoulam, R. Early medical use of cannabis. Nature 1993; 363 : 215.

[5]. Avicenna. The canon of medicine, 1999; Book3, part 1, pp: 65.

[6]. Najmabadi, M. History of medicine in Persian 2000; p.235.

[7]. Karimi, S., Hayatghaibi, H. Effect of Cannabis sativa L. Seed (Hempseed) on Serum Lipid and Protein Profiles of Rat. Pakistan Journal of Nutrition 2006; 5(6): 585-588

[8]. Ayenigbara, G. O. Medical Utility of Cannabis Sativa. IOSR Journal of Pharmacy 2012; 2(3): 460-463

[9]. Maiston, S.A., Galizio, M.G., \& Connors G.J. Drug Use and Abuse (3rd Ed). New York: Harcourt Brace College Publishers. 1999.

[10]. Drugs.com (Internet). Cataflam information from drugs.com; c1996-2014 (Updated 2013-11-18. Cited 2014-04-03). Available from http://www.drugs.com/cataflam.html

[11]. Miller, R. L., Insel, P. A., Melmon, K. L. Inflammatory disorders. In: Melmon KL, Morrelli HF, eds. Clinical Pharmacology: Basic Principles in Therapeutics. New York, NY: Macmillan. 1978; Pp. 657-708.

[12]. Koester, M. C. An Overview of the Physiology and Pharmacology of Aspirin and Non-steroidal Anti-inflammatory Drugs. Journal of Athletic Training 1993 28(3): 252-259.

[13]. European Agency for the Evaluation of Medicinal Products (EMEA) (2003). Diclofenac. Summary report of the Committee for Veterinary medicinal products. http://www.emea.eu.int.

[14]. Ahmed, S. K.Hepatic and renal biochemical responses to the toxicological interaction between Acetylsalicylic acid and diazinon in albino rats. Journal of the Egyptian society of toxicology.2006; 35:1-6

[15]. Okon, V. E., Obembe, A. O., Nna, V. U., Osim, E. E. Long term administration of Cannabis sativa reduces food, water Intake and body weight inmice. International Journal of Science and Research 2014; 3(3): 389-392

[16]. Woolfe, G., MacDonald, A. D. The evaluation of the analgesic action of pethidine hydrochloride (DEMEROL) Journal of Pharmacology and Experimental Therapy 1944; 80:300-307

[17]. Wheeler-Aceto, H., Cowan, A. Standardization of the rat paw formalin test for the evaluation of analgesics. Psychopharmacology 1991;104: 35-44.

[18]. Brown, J. H., Kissel, J. W., Lish, P. M.Studies on the acute inflammatory response. I. Involvement of the central nervous system in certain models of inflammation. Journal of Pharmacology and Experimental Therapy 1968;160: 231-42.

[19]. Lee, I. O., Crosby, G.Halothane effect on formalin-induced paw oedema and flinching in rats. Journal of Korean Medical Sciences 1999; 14: 34-8.

[20]. Coderre, T. J., Vaccarino, A. L., Melzack, R. Central nervous system plasticity in the tonic pain response to subcutaneous formalin injection. Brain Research 1990; 535: 155-8

[21]. Schmidt, K. L., Ott, V. R., Rocher, G., Schaller, H. Heat, cold and inflammation. Z Rheumatology 1979; 38: $391-404$.

[22]. Hong, Y., Abbott, F. V. Behavioral effects of intraplantar injection of inflammatory mediators in the rat. Neuroscience 1994; 63: 827-36.

[23]. Shibata, M., Ohkubo, T., Takahashi, H., Inoki, R. Modified formalin test: characteristic biphasic pain response. Pain1989; 38: 34752 .

[24]. Tjolsen, A., Berge, O. G., Hunskaar, S., Rosland, J. H., Hole, K. The formalintest: an evaluation of the method. Pain $1992 ; 51: 5-17$.

[25]. Voilley, N., Weille, J., Mamet, J., Lazdunski, M.Nonsteroid Anti-Inflammatory Drugs Inhibit Both the Activity and the Inflammation-Induced Expression of Acid-Sensing Ion Channels in Nociceptors. The Journal of Neuroscience 2001; 21(20):80268033

[26]. Edwards, J. A., Oldman, A. D., Smith, L. A., Carroll, D., Wiffen, P. J., McQuay, H. J., Moore, R. A.Oral aspirin in postopera tive pain: a quantitative systematic review. Pain 1999; 81:289-297

[27]. Hasani, A. S., Soljakova, M., Jakupi, M. H., Ustalar-Ozgen, S. Z. Pre-emptive analgesic effect of diclofenac: Experimental study in rats. Middle East Journal of Anesthesiology 2011; 21(3): 355-360

[28]. Torres-López, J. E., Carmona-Díaz, E., Cortés-Peñaloza, J. L., Guzmán-Priego, C. G., Rocha-González, H. I. Antinociceptive synergy between diclofenac and morphine after local injection into the inflamed site. Pharmacological Reports 2013; 65:358-367 